\title{
Le projet pilote de ferme à bilan énergétique positif et les complémentarités productions animales et végétales
}

\author{
Olivier LAPIERRE \\ CEREOPA - AgroParisTech, en collaboration \\ avec Sophie Carton, Dominique Tristant, \\ Yves Python, Bernard de Franssu, \\ Aline Lapierre et Marion Barral \\ 16, rue Claude Bernard, \\ F75231, Paris Cedex 05
}

\begin{abstract}
Since 2006, the "Grignon Energie Positive » programme, based at the experimental farm of AgroParisTech in Grignon, has dealt with the capacity of agriculture to adapt to energy and climate issues. The project has focused on farm system management tools and has enabled to test the environmental efficiency of some farming practices. It has also tackled communication issues associated to changes in farming systems. In 4 years, this project has enabled the achievement of significant improvements in the environmental performance of Grignon Farm, with respect to its fossil energy consumptions and its greenhouse gas emissions. A number of levers for environmental improvement in crop as well as in livestock production have been proven to be efficient.
\end{abstract}

Key words: agriculture, energy, climatic impact environmental performance
C'est durant l'automne 2005 que le prix du pétrole franchissait, pour la première fois depuis la crise des années 1980 la barre des 60 dollars le baril. À l'époque la question soulevée par cette situation était d'ailleurs autant perçue en termes de cherté qu'en termes de rareté. C'est à cette période, qu'à l'initiative du CEREOPA, fut imaginée la mise en place, sur la ferme de l'Agro (devenue AgroParisTech en 2007) à Grignon, du projet Grignon Énergie Positive. Au delà de ce contexte économique particulier, d'autres aspects motivaient ce projet et ont conditionné son déroulement.

\section{À I'origine du projet}

La question de la capacité d'adaptation de l'agriculture et de ses filières aux enjeux de l'énergie et du climat était au cœur du projet. C'est d'ailleurs par analogie au concept de maisons à énergie positive que l'idée du projet a émergé. Dès l'origine du projet, son ambition était de mettre en place, sur la ferme de Grignon, les conditions de la démonstration de l'efficacité des moyens que l'agriculture peut déployer pour affronter ces deux défis. Cette démonstration avait vocation à se dérouler à l'échelle de l'ensemble de l'exploitation et sur une longue durée, au minimum quelques années.

La question des équilibres de l'écosystème n'était, à l'époque, pas la seule préoccupation des porteurs du projet. En 2005, I'agriculture sortait d'une période de 10 années durant laquelle elle avait été régulièrement confrontée au problème de son image dans la société. Dans le prolongement de la crise de la vache folle, I'opinion du grand public à l'égard d'une agriculture parfois considérée comme trop « productiviste » était assez défavorable. Dès le départ, la question de la revalorisation de cette image a imprégné l'esprit du projet. $C^{\prime}$ est avec cette double ambition que le projet fonctionne depuis 2006. Focalisé sur la question des enjeux de l'énergie et du climat, le projet a toujours souhaité ne pas s'enfermer dans une logique purement environnementale. Dès le départ, l'objectif de performance environnementale s'est vu relativisé par la prise en compte simultanée de 2 autres performances : la performance économique et la performance nourricière. C'est dans la logique des $3 \mathrm{P}$ de cette performance (Planète, Profit et Population) que le programme a démarré en 2006 et fonctionne depuis. II semblait à l'époque et il continue de sembler à ses auteurs que la démonstration du bienfondé de certaines solutions à l'égard de l'environnement ne pouvait pas être conduite sans que, dans le même temps, on s'interroge sur leurs conséquences sur la performance économique et sur le potentiel nourricier de l'exploitation. Leur acceptabilité par les agriculteurs imposait cette triple vision (figure 2).

L'installation du projet sur la ferme de Grignon lui a permis de profiter d'un écosystème très favorable (encadré 1). Son lancement a été rendu possible par la mobilisation d'un pool de partenaires qui lui ont apporté leur soutien et leur éclairage.

C'est sur ces bases que le projet a été effectivement lancé en 2006. Son « acte 1 » s'est déroulé jusqu'en 2009. Le présent article en présente la démarche et les résultats. Depuis 2010, son « acte 2 » a pris le relais. Avec le soutien de la majorité des partenaires initiaux et avec celui de nouveaux partenaires, il envisage aujourd'hui un élargissement du champ des préoccupations et une extension de son aire d'application.

\section{Diagnostic initial et choix d'orientation}

Pour engager la ferme dans la voie de la performance environnementale, la réalisation d'un diagnostic et le repérage des leviers de progrès constituaient une première étape indispensable. C'est ce qui a été fait à Grignon dès 2006, en s'appuyant sur les résultats 2005 du fonctionnement de l'exploitation.

\section{Diagnostic énergétique et climatique}

La question du choix d'une méthode de diagnostic s'est posée dès le départ. De nombreuses solutions sont proposées dans la littérature. Vingt-quatre outils et méthodes d'analyse de la durabilité environnementale et/ou économique d'une exploitation agricole avaient été recensés à l'époque (France et International). Pour des raisons d'adéquation aux objectifs du projet (énergie et gaz à effet de serre) et pour sa capacité à quantifier les phénomènes, $c^{\prime}$ est la méthode Planète ${ }^{\circledR}$, développée par Solagro qui a été retenue. Une application Excel construite en interne, permettant d'élargir les diagnostics énergie et effet de serre aux activités non agricoles stricto sensu de l'exploitation (transformation du lait dans la laiterie, bâtiments administratifs). Ce tableur Excel a été construit en se basant sur la méthodologie du Bilan Carbone ${ }^{\circledR}$ et en utilisant des données sources (facteurs de conversion en équivalent 
énergie et effet de serre) provenant du Bilan Carbone $^{\circledR}$ et de Planète $^{\circledR}$. La méthode Dialecte $^{\circledR}$ (Solagro) avait également été retenue pour la réalisation d'un diagnostic environnemental plus complet. Dans le contexte du projet, elle a finalement été abandonnée, son diagnostic s'étant révélé insuffisamment précis pour révéler des voies de progrès.

\section{Résultats}

Les résultats obtenus à l'époque (figure 3) ont permis d'identifier les postes les plus impactants sur le plan environnemental.

- En matière de consommation d'énergie fossile il est apparu que les 408 tep (tonnes équivalent pétrole) consommées en 2005 étaient largement dues à une consommation « indirecte » résultant des achats d'engrais $(29,6 \%)$ et des achats d'aliments du bétail (18,4\%). II est également ressorti de ce diagnostic que la consommation de fioul sur l'exploitation représentait également un poste significatif de dépense (22,6\%). Les activités relatives à l'activité agricole au sens strict du terme représentaient plus de $80 \%$ de l'énergie fossile consommée. La transformation du lait en représentait un peu moins de $10 \%$.

- En matière de gaz à effet de serre, les émissions totales de la ferme en 2005 étaient de 2627 tonnes d'équivalent CO2. Sur ce total, les activités relatives à l'activité agricole au sens strict du terme représentaient $88 \%$, la transformation du lait $8 \%$. Au niveau de cet impact climatique, il est intéressant de remarquer l'importance de la contribution des émissions de méthane ( $\mathrm{CH} 4)$ par les ruminants et de celle de protoxyde d'azote (N2O) par les cultures. Ces ceux sources, émises «directement» par l'exploitation, représentent $42 \%$ de son pouvoir de réchauffement global. Cette situation illustre parfaitement la spécificité du secteur agricole à l'égard de l'enjeu climatique. En agriculture en effet, le couplage habituellement constaté entre consommation d'énergie fossile et émissions de gaz à effet de serre est loin d'être aussi net qu'il l'est dans d'autres secteurs d'activité.

Pour éviter de réduire la question de la performance de l'exploitation agricole à celle de ses impacts environnementaux et pour engager un discours équilibré avec le public sur cette question, la solution a été de communiquer en même temps sur sa performance nourricière. La balance de la figure 4 est dont devenue une illustration emblématique la problématique du projet Grignon Énergie Positive. Elle explicite clairement l'enjeu du projet qui consiste bien à tenter de limiter au maximum les impacts énergie et climat de l'activité agricole sans remettre en cause sa vocation première qui reste de nourrir les hommes.

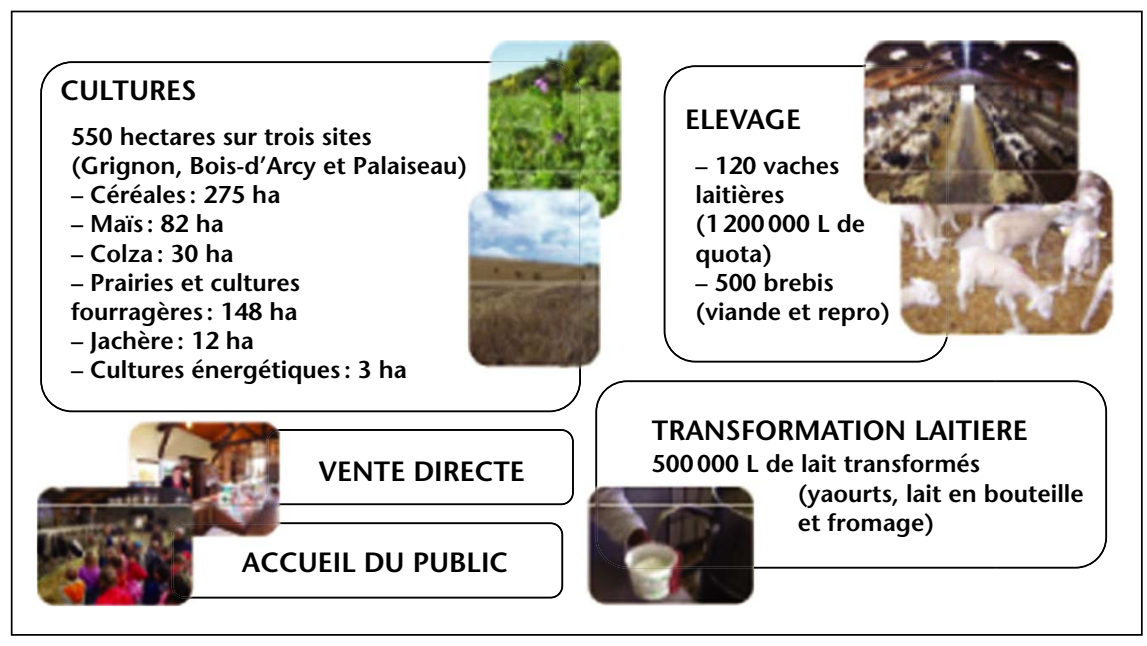

Figure 1. La ferme de Grignon.

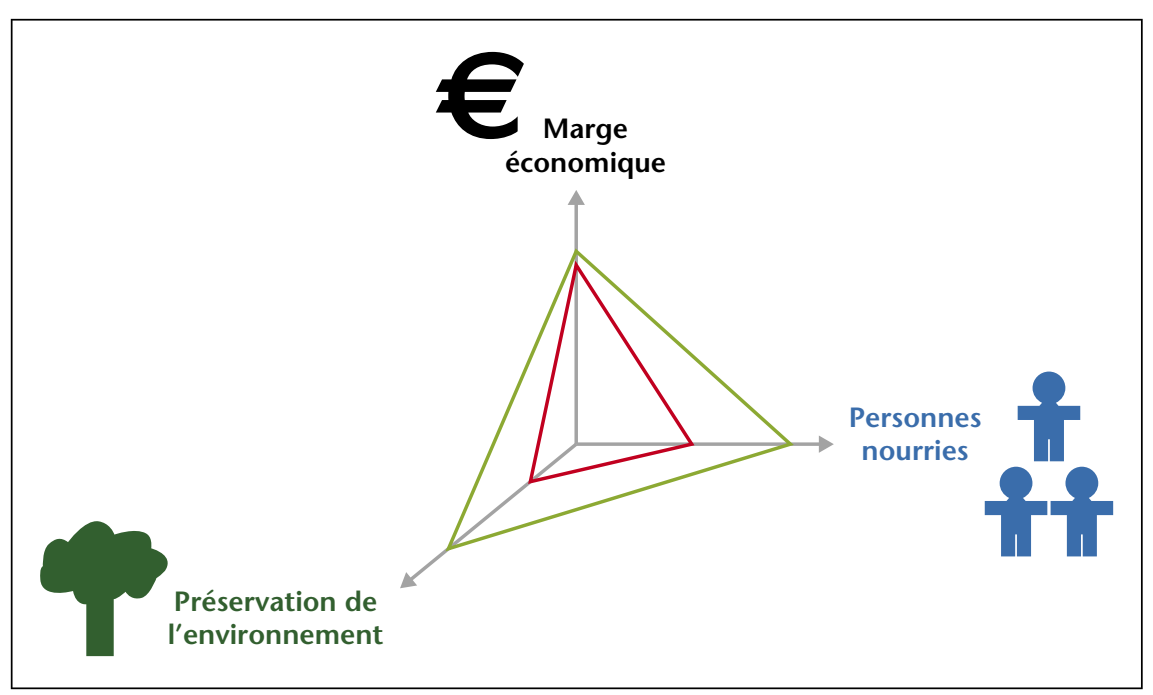

Figure 2. Le triangle des performances du projet GE+.

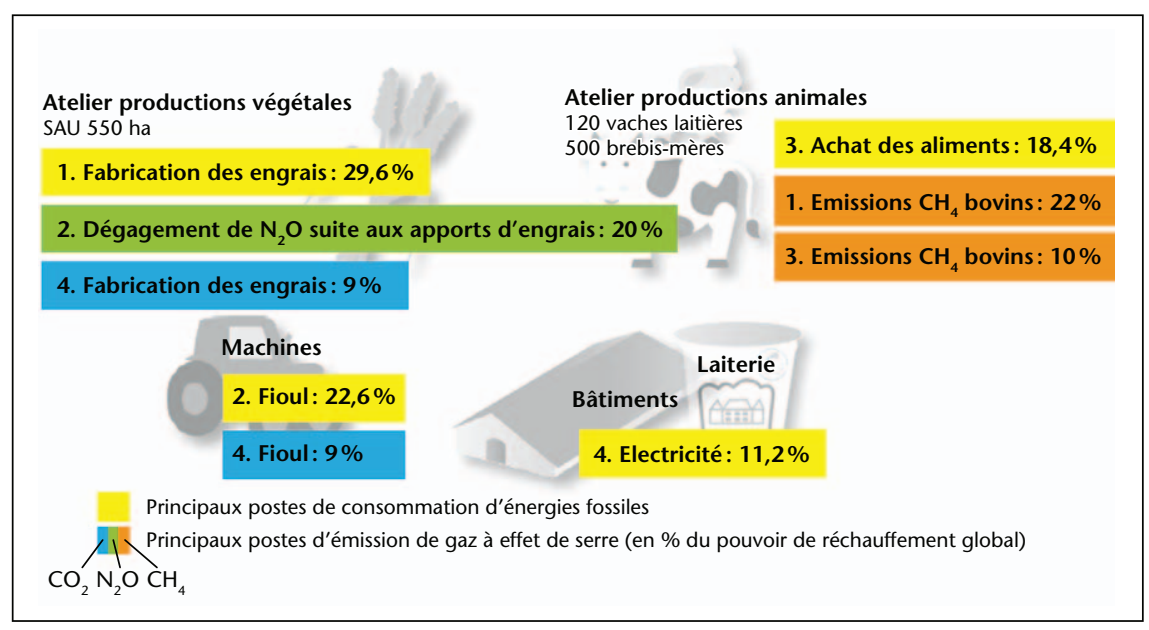

Figure 3. Les résultats du diagnostic énergie et effet de serre de la ferme de Grignon en 2005. 
Limites

Même si elle nous a permis d'obtenir une vision précise des impacts des activités mises en œuvre sur la ferme de Grignon, la démarche de diagnostic utilisée à l'époque a aussi montré quelques limites. Trois d'entre elles nous semblent devoir être mentionnées.

- La première est relative au caractère encore incertain des hypothèses mobilisées dans les calculs qui permettent d'estimer les impacts des activités. Réalisés sur la base des principes des méthodes d'ACV, ces calculs utilisent des coefficients qui permettent de transformer les données de flux élémentaires repérés dans le fonctionnement du système de production en impacts environnementaux induits. C'est ainsi que l'on passe d'une quantité d'engrais azoté utilisée à une évaluation de son impact climatique par la prise en compte des émissions de dioxyde de carbone et de protoxyde d'azote engendrées durant l'étape de sa production industrielle et des émissions de protoxyde d'azote associées à sa dynamique dans le sol. La fiabilité de ces coefficients reste un élément sensible de I'analyse. Les valeurs utilisées en 2006 pour estimer les impacts de l'année 2005 ont d'ailleurs été en partie remises en cause depuis. Les résultats les plus récents (cf. infra) ont été calculés sur la base de valeurs différentes de celles mises en œuvre en 2006 et ont imposé un exercice de rétropolation seul garant de la cohérence des dynamiques constatées sur la ferme.

- La deuxième concerne la variabilité des phénomènes impliqués dans ces impacts énergétiques et climatiques. D'une année sur l'autre, les résultats sont susceptibles de varier de façon assez significative même en l'absence de modification significative des pratiques. C'est ce qui a été constaté sur la ferme de Grignon entre 2005 et 2006. Le calcul effectué sur la base de ce qui s'était passé en 2006 a effectivement révélé une diminution de près de $25 \%$ des consommations d'énergie fossile de la ferme et de ses émissions de gaz à effet de serre par rapport à la situation de 2005. Or, aucun changement significatif des pratiques n'avait été encore mis en place. L'explication de cette apparente amélioration de la performance environnementale est principalement due aux conditions climatiques de I'hiver 2005-2006. En laissant dans le sol des niveaux de résidus azotés élevés, elles ont permis de limiter significativement des épandages d'engrais azotés en 2006 et ont ainsi contribué à une réduction significative des impacts. Cet exemple illustre bien la nécessité d'introduire le temps long dans l'interprétation des dynamiques de progrès que seront susceptibles de mettre en place les agriculteurs.

102 habitants 440 habitants

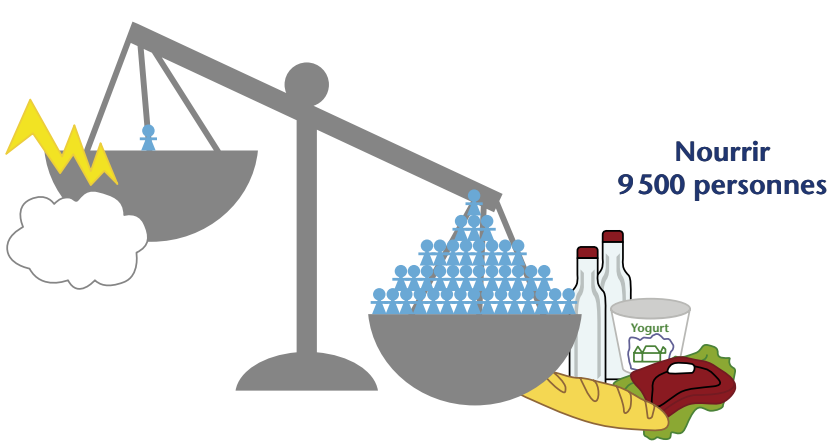

La ferme de Grignon en 2005

Figure 4. Un discours équilibré sur les enjeux de performance environnementale de l'agriculture.

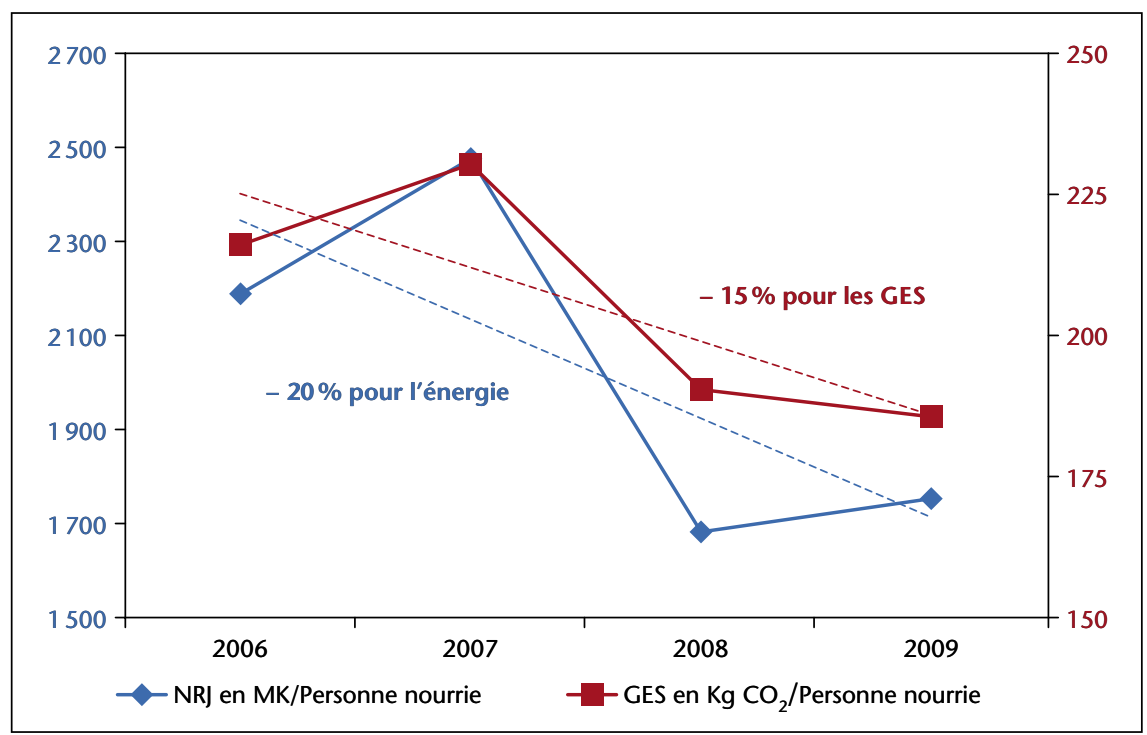

Figure 5. Évolution de la performance énergétique et climatique de la ferme de Grignon entre 2006 et 2009.

- La troisième concerne le caractère statique de ces approches. Ces diagnostics sont efficaces pour fournir une photographie de la situation des exploitations agricoles à un instant donné. Par contre, ils ne permettent pas d'obtenir une vision dynamique des situations et présentent donc certaines limites pour juger de la pertinence des différentes voies de progrès qui s'offrent à une exploitation ainsi que pour juger, dans le temps, de la pertinence des mesures mises en œuvre pour améliorer leur performance. C'est une raison qui a justifié le recours à une solution plus adaptée : la mise en œuvre de l'outil PerfAgro P3.

C'est à partir des explorations réalisées à travers cet outil que les premières pistes $d^{\prime}$ amélioration ont pu être dégagées et c'est à partir de cette période que les améliorations de la performance environnementale de la ferme peuvent être analysées.

\section{Les voies de la performance environnementale}

Repérées en 2006, les mesures susceptibles d'améliorer la performance environnementale de la ferme de Grignon ont été progressivement mises en place à partir de cette date. Avec 4 années de recul et en considérant la situation de l'année 2006 comme base de départ on peut déjà mesurer les progrès qui ont été réalisés sur l'exploitation en matière de consommation d'énergie fossile et d'émission de gaz à effet de serre.

\section{Sur l'ensemble de l'exploitation}

Mesurés sur l'ensemble de l'exploitation agricole, y compris I'activité de transformation laitière) les progrès enregistrés depuis 2006 sont assez significatifs. En rapportant les impacts environnementaux au nombre de 


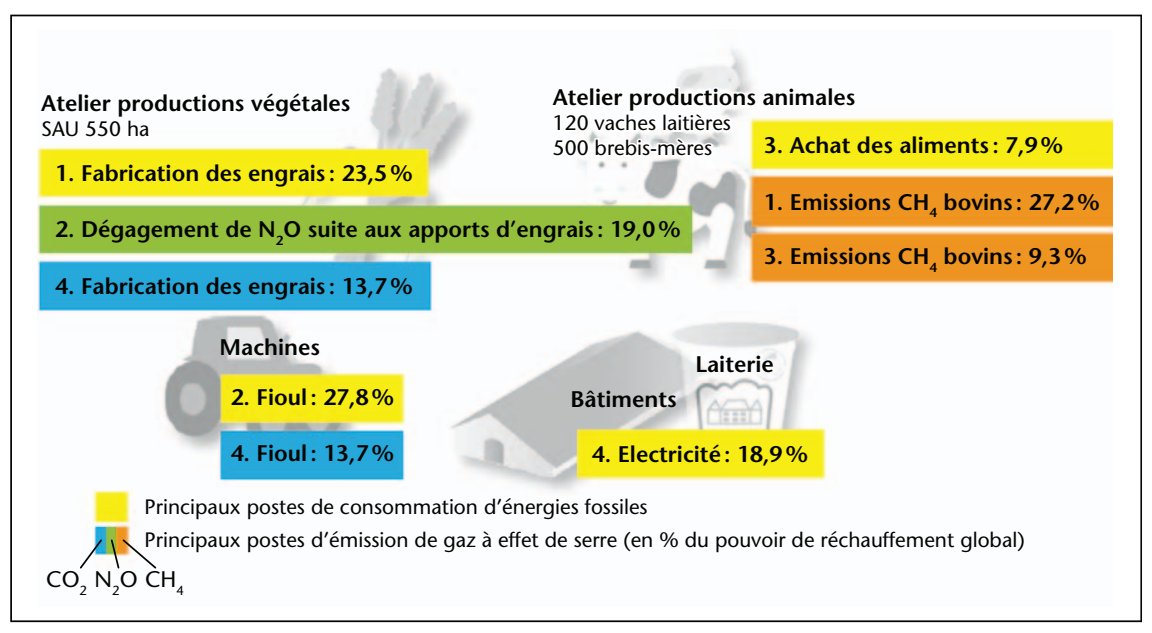

Figure 6. Les résultats du diagnostic énergie et effet de serre de la ferme de Grignon en 2009.

personnes potentiellement nourries sur l'exploitation (calcul effectué sur la base de l'énergie alimentaire), on peut constater une amélioration de $20 \%$ de l'empreinte énergétique et de $15 \%$ de l'empreinte climatique (figures 5 et 6). Cette évolution, même si elle résulte d'une dynamique irrégulière, traduit bien les bénéfices des adaptations mises en œuvre sur la ferme de Grignon depuis 2006.

\| est intéressant de remarquer que cette amélioration est principalement liée à une amélioration de l'efficience du système. En effet, si I'on considère les niveaux totaux des consommations d'énergie fossile et des émissions de gaz à effet de serre, on constate qu'ils n'ont que faiblement diminué pour les premiers $(-3,48 \%)$ et qu'ils ont même très légèrement augmenté pour les seconds $(+2,65 \%)$. C"est donc bien la capacité du système de production à assurer une offre agricole en hausse $(+20,4 \%$ sur la base $d u$ nombre de personnes nourries) tout en limitant l'évolution de ses impacts qui est à l'origine de cette amélioration de sa performance environnementale globale. Cette transformation de la performance globale de l'exploitation se traduit également par un rééquilibrage du poids respectif des différents postes qui participent de ce bilan (figure 7).

Au sein de cette globalité, la situation des différentes activités est très variable. La performance s'est beaucoup améliorée sur les ateliers

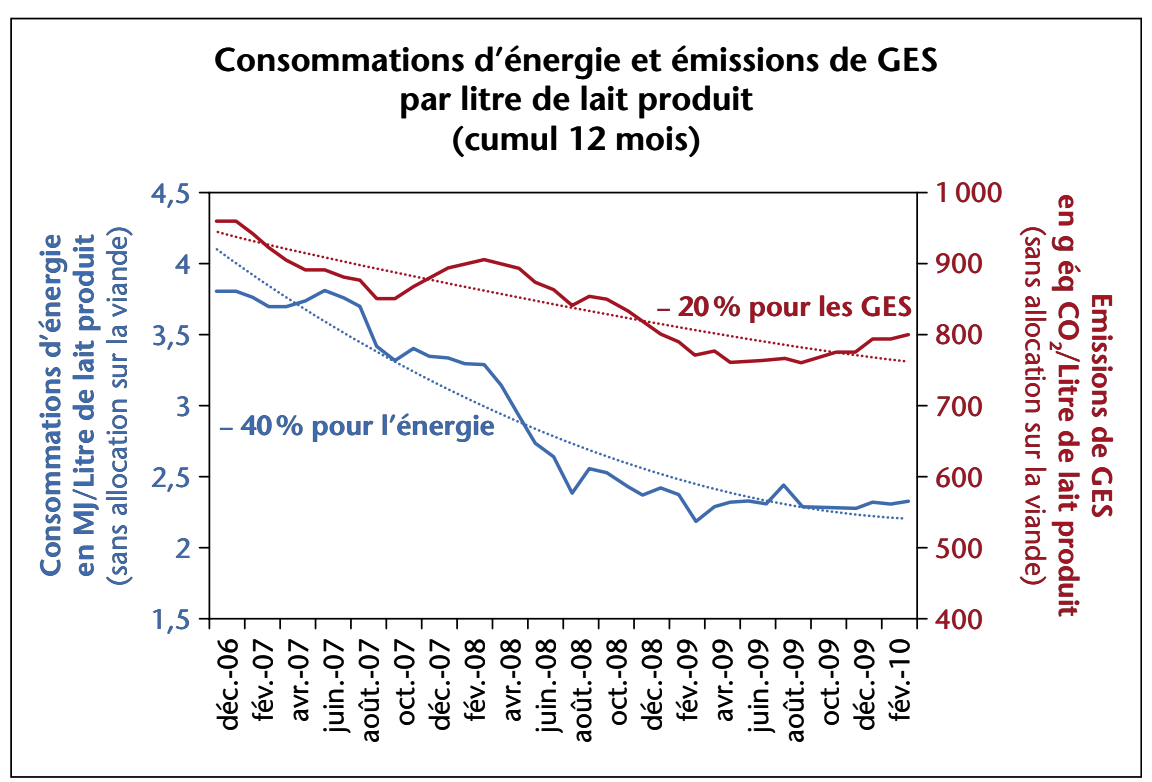

Figure 7. Évolution de la performance énergétique et climatique de l'atelier laitier de la ferme de Grignon entre 2006 et 2009. de production animale (bovins laitiers et ovins viande) alors qu'elle s'est plutôt un peu dégradée sur l'atelier de production végétale (cultures de vente).

Ces résultats globaux doivent cependant être interprétés en considérant qu'en 2009 tous les changements de pratiques mis en œuvre à Grignon n'avaient pas encore porté leurs fruits. $C^{\prime}$ est en particulier le cas des changements d'assolement qui se sont traduits, en 2010, par la création de 7 hectares d'herbe destinés au pâturage des vaches laitières et de 14 hectares de féverole et par l'augmentation de 25 hectares de la surface cultivée en luzerne.

\section{Au niveau de la production laitière}

L'amélioration de la performance environnementale de la production laitière constitue certainement le résultat le plus spectaculaire de ceux qui ont été obtenus à Grignon depuis 2006.

La figure 7 permet d'apprécier la façon dont ont évolué les 2 critères de performance suivis sur le troupeau de Grignon depuis 2006. En trois ans c'est à une réduction d'environ $40 \%$ de l'énergie fossile consommée et à une diminution d'environ $20 \%$ des émissions de gaz à effet de serre qu'on est arrivé. Ces améliorations sont très significatives et démontrent bien l'importance des marges de progrès qui peuvent exister sur une exploitation même sans en bouleverser le système de production. De l'ensemble des facteurs qui ont contribué à cette amélioration, on peut en retenir principalement deux.

- Le premier est celui de la stratégie d'alimentation du cheptel. La diminution de l'utilisation de produits déshydratés au profit de fourrages produits sur l'exploitation explique une bonne part de l'amélioration constatée. Cela est particulièrement vrai sur le poste d'impact énergétique.

- Le second est la productivité du cheptel laitier. Depuis 2006, des efforts ont été faits pour continuer d'améliorer le potentiel de production du cheptel et pour limiter au maximum les facteurs d'improductivité par une plus grande maîtrise de l'état sanitaire et par l'amélioration des performances de reproduction.

Par ailleurs, une politique de valorisation optimale des effluents d'élevage représente également un gisement intéressant de performance. En cédant aux cultures de vente les effluents non utilisés pour la fertilisation des surfaces fourragères, le troupeau laitier est à l'origine d'une économie sur les achats d'engrais. Traduite en terme d'énergie fossile économisée, celle qui aurait été nécessaire à la fabrication des engrais ainsi économisés, cela représente $30 \%$ des dépenses énergétiques engagées par ailleurs sur l'ensemble de l'atelier laitier. Cette situation 
montre bien l'avantage trouvé dans cette complémentarité entre cultures de vente et productions animales.

La stabilisation de la performance depuis 2009, voire sa légère dégradation, peuvent traduire le fait qu'il sera sans doute difficile de poursuivre dans le sens d'une réduction des émissions des impacts. La mesure des bénéfices de l'augmentation des surfaces fourragères en 2010 n'est pas encore perceptible. De toute façon elle ne pourra s'apprécier qu'à la mesure des effets qu'elle risque d'avoir sur le potentiel nourricier de l'exploitation. Les surfaces engagées dans la production de fourrage sont autant de surfaces retirées de la production des cultures des ventes dont le potentiel nourricier n'est plus valorisé.

Même si des marges de progrès existent encore au niveau de l'activité laitière de la ferme de Grignon, il semble désormais utile de rechercher d'autres moyens que ceux de l'atténuation de ses impacts. La voie de la compensation est aujourd'hui considérée avec une attention particulière. Plusieurs pistes ont été explorées et évaluées pour Grignon. II s'agit de celles de la production d'énergie, soit par le biais de la culture de plantes énergétiques (miscanthus et switchgrass), soit par le biais de l'investissement en panneaux photovoltaïques, soit par le biais de la production de biogaz. C'est finalement la dernière voie qui a été privilégié avec l'ambition d'atteindre une situation où la ferme devrait pouvoir s'afficher comme ferme à énergie positive (restituant à la collectivité plus d'énergie que celle qu'elle consomme pour son fonctionnement) et comme ferme neutre en carbone.

\section{Au niveau de la production végétale}

L'évolution des impacts environnementaux des cultures de vente s'est montrée moins régulière que celle des impacts de la production laitière (figures 8 et 9). Le caractère particulièrement favorable de l'année 2006 (cf. infra), prise comme année de référence, explique en partie cette situation. Face à cette irrégularité initiale, les résultats des années 2008 et 2009 semblent indiquer une meilleure maîtrise de la situation. II n'en demeure pas moins que l'interprétation de résultats "par culture » reste délicate. Deux éléments au moins expliquent cette difficulté.

- Le premier est inhérent à l'activité de production végétale. C'est celui de la variabilité interannuelle des rendements. Significatif même à une échelle géographique large les effets de cette variabilité sont très importants à l'échelle d'une exploitation agricole. D'une année à l'autre, les fluctuations non anticipées du rendement sont susceptibles de détériorer les performances. La figure 10 illustre cet aspect à

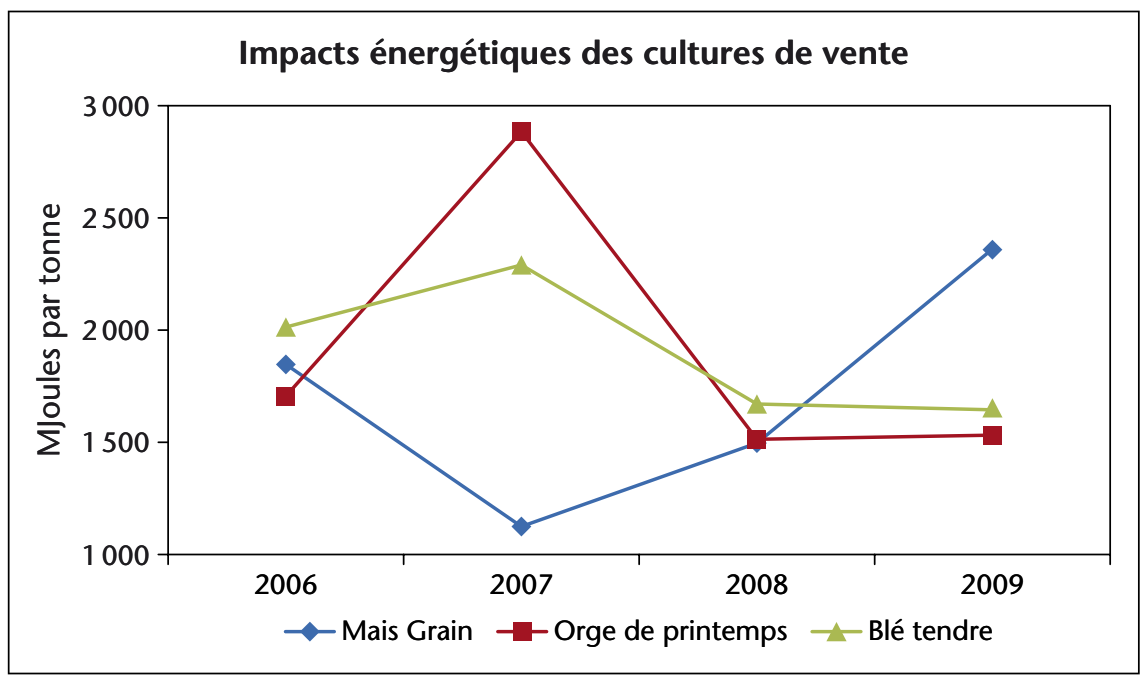

Figure 8. Evolution De La Performance Energetique Et Climatique De L'atelier Cultures de vente de la ferme de Grignon entre 2006 et 2009.

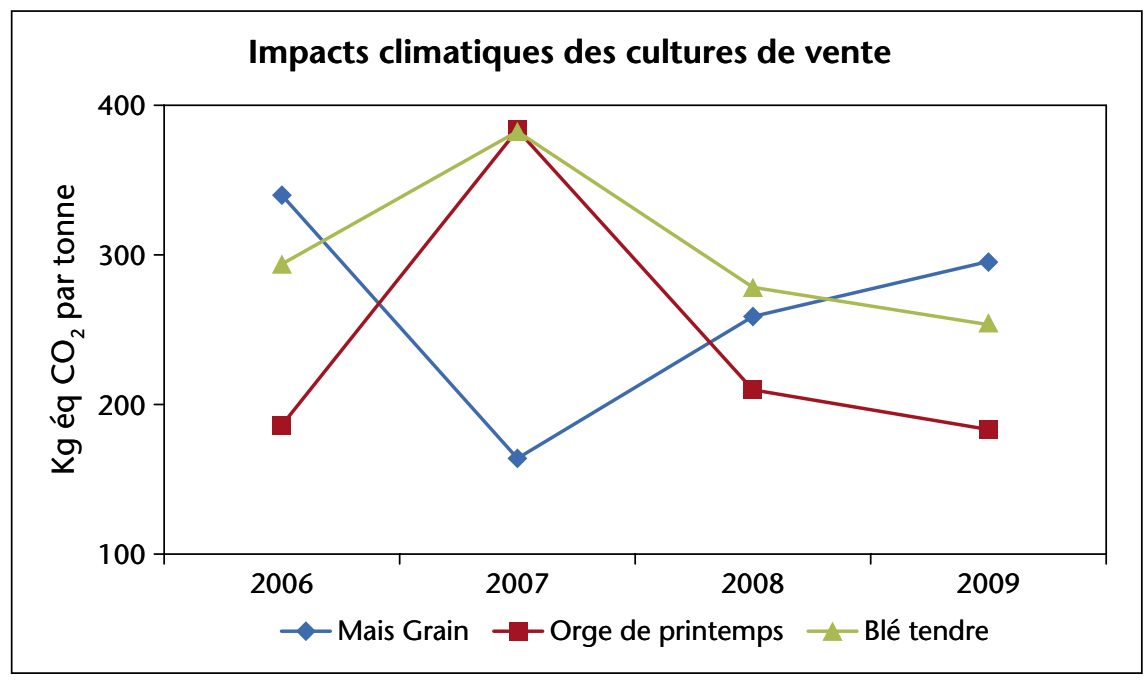

Figure 9. Évolution de la performance énergetique et climatique de l'atelier cultures de vente de la ferme de Grignon entre 2006 et 2009.

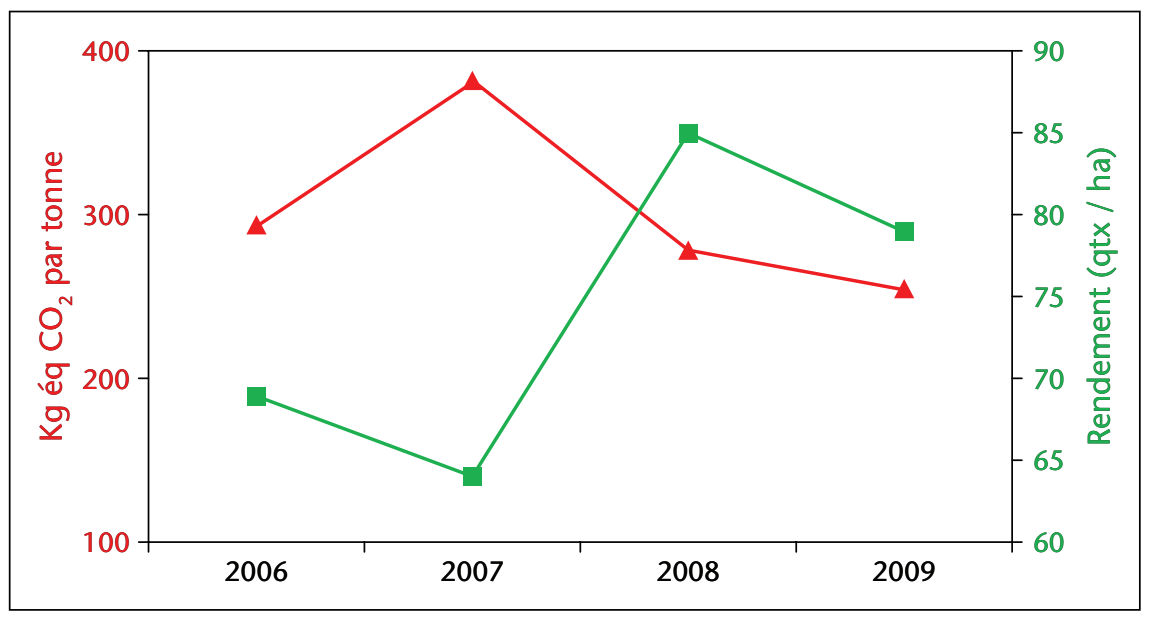

Figure 10. Émissions de GES et rendements du Blé sur la ferme de Grignon entre 2006 et 2009. 


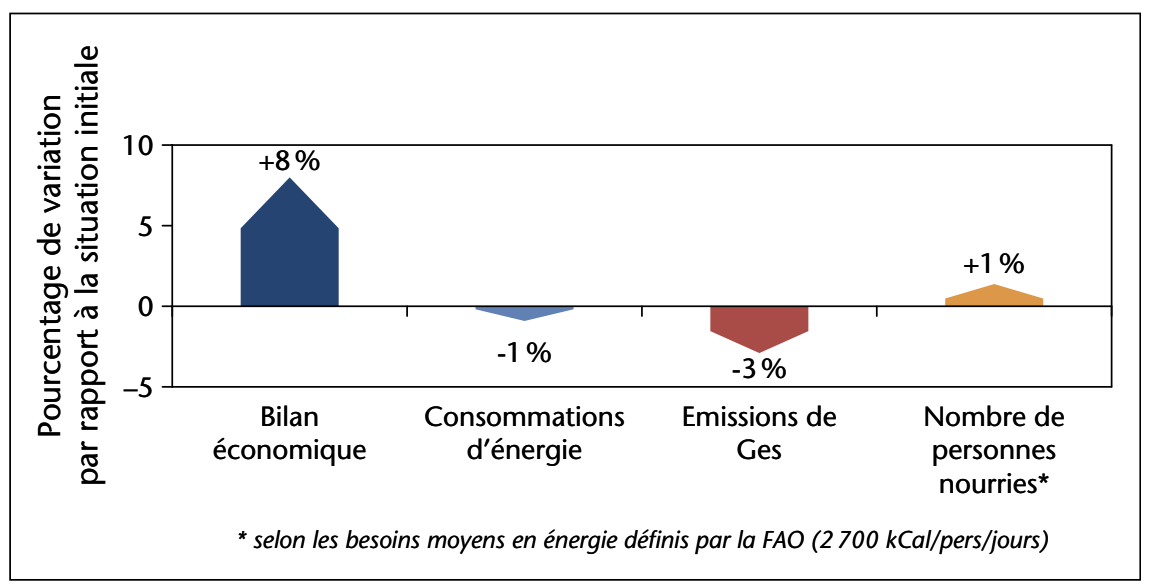

Figure 11. Bénéfices à l'échelle de l'exploitation d'un développement des cultures de colza et de féverole.

nes nourries selon les besoins en énergie augmente, et ce grâce à la vente de colza et de féverole.

Cet exemple illustre bien la nécessité de sortir d'une approche trop linéaire quand on souhaite évoquer la performance environnementale des activités agricoles. II est évident que plusieurs des facteurs qui la déterminent sur une exploitation relèvent des interactions entre les activités. Dans le cas présent, les interactions entre cultures au sein d'une rotation limitent les besoins de fertilisation sur l'ensemble de l'assolement. Les interactions entre activités animales et activités végétales se jouent sur des questions de fertilisation organique et de bilan paille de l'exploitation.

\section{Conclusion}

partir de la culture de blé. C'est en particulier le cas de l'année 2007 pour laquelle un rendement dégradé, inférieur à 65 quintaux pour le blé, explique largement la médiocre performance environnementale.

- Le second aspect est celui de la variabilité spatiale du rendement. Selon les parcelles de l'exploitation les rendements d'une même année peuvent présenter des écarts significatifs. Ce phénomène est encore accentué à Grignon où le potentiel des terroirs exploités est assez variable. Ainsi, une part des écarts de rendement d'une année à l'autre est imputable aux modifications de l'assolement et au changement de localisation de la culture.

Une exploration plus approfondie de cette double variabilité, temporelle et spatiale, permet de repérer l'effet d'un déterminant majeur. C'est l'efficacité de la fertilisation qui semble conditionner le plus la performance environnementale des cultures. Les écarts constatés entre parcelles et/ou entre années sont étroitement liés au niveau du bilan $\mathrm{N}$ qu'il est possible de calculer. Manifestement, l'optimisation de la fertilisation azotée apparaît comme le levier principal de la performance environnementale. Le suivi des reliquats azotés, la prévision du rendement et les solutions de sécurisation de ce rendement sont les pratiques les plus susceptibles de garantir la performance énergétique et climatique des cultures.

\section{L'approche « système de production »}

Même si elle permet d'obtenir des informations utiles, une décomposition analytique poussée de l'impact environnemental ne suffit pas à garantir la performance de l'exploitation agricole. Le détour par l'approche du système de production est tout à fait essentiel. Cette affirmation peut facilement être illustrée à partir des résultats de la ferme de Grignon.

En 2009, dans la perspective de la réforme de la PAC, plusieurs options de production ont été étudiées à Grignon. Les pistes explorées étaient (1) l'accroissement de la surface cultivée en féverole, (2) la mise en place de cultures de colza derrière légumineuses, (3) l'accroissement des surfaces en herbe et l'accès au pâturage des vaches en seconde partie de lactation et (4) le développement du mélange luzerne triticale. L'intérêt potentiel de ces options de production était justifié par la perspective $d^{\prime}$ effets favorables pour lesquels il était possible deformuler des hypothèses quantifiées. Ce sont ces hypothèses qui ont alors été utilisées pour explorer les bénéfices potentiels, à l'échelle de l'exploitation agricole, de la mise en œuvre de ces changements.

La figure 11 donne un exemple d'une des solutions testées. II s'agit de celle qui explorait l'intérêt de l'introduction de 25 ha de féverole et de 70 ha de colza pris en majorité sur les surfaces en maïs grain (réduites à $0 \mathrm{ha}$ ), mais aussi celles en blé. Les gains de performance économique sont essentiellement dus à l'augmentation des ventes de cultures, mais également à la diminution des dépenses liées aux achats d'engrais minéral. En revanche le coût des aliments achetés augmente, dû à l'achat de paille pour les litières des animaux. La diminution des consommations d'énergie et des émissions de gaz à effet de serre est relativement faible et est principalement due à la diminution de l'utilisation d'engrais. Le nombre de person-
Quatre années de recul ont permis de mieux cerner la question de la performance énergétique et climatique de l'activité agricole sur la ferme de Grignon. Elles ont abouti à la mise en place de solutions de suivi de cette performance et à la conception d'outils d'aide à la décision susceptibles de guider les agriculteurs dans l'exploration de solutions plus vertueuses et dans leur mise en œuvre sur leur exploitation. Elles ont aussi permis de faire valoir l'intérêt de leviers qui se sont avérés efficaces pour contribuer à réduire l'impact environnemental de la ferme sans remettre en cause sa capacité nourricière ni sans compromettre sa performance économique. Elles ont confirmé l'intérêt de l'association d'une activité de production animale à celle de production végétale. Elles ont permis de démontrer que la productivité restait un levier très efficace en matière de performance environnementale. Elles ont aussi permis de mesurer les bénéfices potentiels de nouvelles associations végétales où les légumineuses et les oléagineux avaient toute leur place.

La poursuite de la démonstration sur d'autres terrains que celui de Grignon et avec des préoccupations environnementales complémentaire de celles de l'énergie fossile consommée et des émissions de gaz à effet de serre permettra de compléter l'approche. Cela devrait contribuer à améliorer l'éclairage des agriculteurs sur les possibilités qu'ils ont de s'engager sur la voie d'une agriculture plus vertueuse et $d^{\prime}$ en faire un atout, valorisable et communicable, pour mieux répondre aux attentes des consommateurs et aux aspirations de leurs concitoyens. 


\section{Encadré 1 \\ Aux portes de Paris, une plate-forme de démonstration sur la performance environnementale de l'agriculture du $X X I^{e}$ siècle}

\section{Grignon Énergie Positive : un écosystème favorable}

En installant ce projet à Grignon, AgroParisTech et le Céréopa ont eu l'ambition de potentialiser les synergies qui pouvaient s'y développer. Cela a d'abord concerné le potentiel humain. Incontestablement, une école d'ingénieur, par la diversité et la complémentarité de ses enseignants et chercheurs, dispose d'atouts favorables pour piloter un tel projet. Très vite, s'est constituée une équipe dont les membres ont permis de disposer de l'ensemble des compétences nécessaires. À côté des responsables et salariés de l'exploitation agricole, habitués à la conduite d'une exploitation agricole confrontée aux rigueurs de l'expérimentation, c'est une équipe composée de zootechniciens, d'agronomes, de spécialistes des questions d'économie de l'exploitation agricole, de spécialistes du climat, de biologistes qui a permis de mobiliser l'expertise nécessaire à la prise en compte de tous les aspects liés à une question aussi complexe. C'est en particulier la proximité d'équipes de recherche réputées sur les questions de l'interface production végétale-climat ainsi que sur celles de I'alimentation des vaches laitières à haut niveau de production qui aura permis de mettre en place des dispositifs de production de références originaux à l'époque et aujourd'hui reconnus. La mesure des émissions de protoxyde d'azote par les cultures et celle des émissions de méthane entérique par les vaches laitières qui sont aujourd'hui mises en œuvre en routine sur la ferme sont l'illustration des avantages tirés de cet environnement scientifique remarquable.

Cela a aussi concerné les caractéristiques du système de production de la ferme de Grignon (figure 1).

Sur une superficie, à l'époque de 550 hectares, sont associées des activités de grandes cultures (blé, orge, colza, maïs) et des activités d'élevage (bovins lait et ovins viande). Joint à cela, le fait que la ferme exploite 3 territoires assez différents dans le sud et l'ouest de Paris permet de disposer d'une variété de situations intéressante. En soi, le fait de disposer d'une association production animale-production végétale est aussi un atout car il permet d'évaluer objectivement l'intérêt potentiel d'une combinaison qu'on présente volontiers comme vertueuse en matière d'environnement.

Outre la diversité des productions agricoles, la ferme de Grignon a également la caractéristique d'être engagée dans la transformation de son lait en produits finis. Le conditionnement de lait et sa transformation en crème, fromage blanc et yaourt, permettent de prolonger les approches du maillon production tout au long de la filière. En poussant l'étude des problèmes jusqu'au stade du produit fini, celui que perçoit le consommateur, on dispose d'un levier de communication tout à fait appréciable. À une période où la question de l'étiquetage environnemental des produits de consommation est mise sur le devant de la scène, cela représente un indéniable intérêt.

Le dernier élément est aussi une caractéristique de l'exploitation agricole. Située à 30 km à l'ouest de Paris, la ferme de Grignon s'est donnée depuis plus de 10 ans une vocation d'accueil du public. La création d'un circuit du lait, en partenariat avec le CIDIL, lui permet de recevoir chaque année plus de 10000 visiteurs parmi lesquels 4000 scolaires.

\section{Les partenaires du projet Grignon Énergie Positive}

Entre 2006 et 2009, le projet Grignon Énergie Positive a profité du soutien des partenaires suivants :

Arvalis - CETIOM - Sofiprotéol - CNIEL - Crédit Agricole - Ademe Suez Environnement - Conseil Régional Île de France - Conseil Général des Yvelines

\section{L'outil PerfAgro P3}

PerfAgro P3 est un outil d'aide à la décision fondé sur la mise en œuvre des techniques d'optimisation. II procède de l'application de la technique de calcul de programmation linéaire à un modèle du fonctionnement de l'exploitation agricole. Son objectif est d'aider à déterminer les caractéristiques de fonctionnement qui permettent d'optimiser la performance de l'exploitation agricole.

En permettant de travailler sur plusieurs critères d'optimisation, il permet d'aborder la question de la performance de l'exploitation en intégrant les 3 enjeux (les 3P) devenus incontournables que sont :

- la performance économique (Profit), abordée sous I'angle d'une « marge PerfAgro » qui constitue un indicateur de la rentabilité de l'exploitation agricole ;

- la performance environnementale (Planète), abordée sous l'angle des enjeux énergie et gaz à effet de serre ;

- la performance nourricière (Population), abordée sous l'angle de la satisfaction des besoins nutritionnels de la population en énergie et en protéine.

La prise en compte simultanée de ces trois catégories d'enjeu (figure 2) est destinée apporter une réponse adaptée au contexte dans lequel se pose aujourd'hui les questions sur l'adaptation des systèmes de production agricole et qui se caractérise par plusieurs tendances fortes :

- réévaluation des politiques agricoles qui mettent les agriculteurs face à de nouveaux équilibres de prix et à un moindre encadrement des marchés ;

- instabilité des marchés qui contraint à mieux évaluer les options de production en particulier en ce qui concerne leur risque ;

- émergence des politiques environnementales (Ecophyto, affichage environnementale, HVE, etc.) qui instaurent progressivement de nouvelles contraintes et imposent de réviser, au moins partiellement, les options techniques de production ;

- mise en place d'un plan de performance énergétique destiné à amener un fort pourcentage des exploitations agricoles vers une plus grande autonomie énergétique ;

- croissance démographique et évolution des modes de consommation des populations des pays émergents qui remet sur le devant de la scène l'enjeu de la production et pose la question de la capacité de l'agriculture à « nourrir le monde ».

Ce modèle est construit de façon à permettre une prise en compte détaillée du fonctionnement global de l'exploitation. II permet de décrire ce fonctionnement à partir des éléments qui le caractérisent en distinguant, le cas échéant, plusieurs zones sur l'exploitation agricole. 